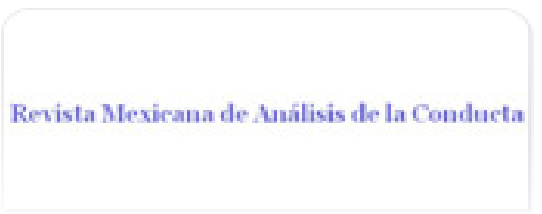

Revista Mexicana de Análisis de la Conducta ISSN: 0185-4534

editora@rmac-mx.org

Sociedad Mexicana de Análisis de la Conducta

México

Padilla Vargas, María Antonia; Fernández Sordo, Gonzalo Efectos de manipular características textuales del referente en la lectoescritura de textos científicos Revista Mexicana de Análisis de la Conducta, vol. 40, núm. 3, diciembre, 2014, pp. 47-71

Sociedad Mexicana de Análisis de la Conducta Distrito Federal, México

Disponible en: http://www.redalyc.org/articulo.oa?id=59335812004

- Cómo citar el artículo

Número completo

* Más información del artículo

- Página de la revista en redalyc.org

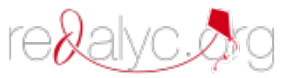

Sistema de Información Científica

Red de Revistas Científicas de América Latina, el Caribe, España y Portugal Proyecto académico sin fines de lucro, desarrollado bajo la iniciativa de acceso abierto 


\title{
EFECTOS DE MANIPULAR CARACTERÍSTICAS TEXTUALES DEL REFERENTE EN LA LECTOESCRITURA DE TEXTOS CIENTÍFICOS
}

EFFECTS OF VARYING TEXTUAL CHARACTERISTICS OF THE REFERENT ON READING AND WRITING SCIENTIFIC TEXTS

\section{MARÍA ANTONIA PADILLA VARGAS Y GONZALO FERNÁNDEZ SORDO CENTRO DE ESTUDIOS E INVESTIGACIONES EN COMPORTAMIENTO UNIVERSIDAD DE GUADALAJARA}

\begin{abstract}
Resumen
Se evaluó el efecto de variar las características textuales de artículos científicos (volviéndolos no estructurados), sobre el nivel funcional (intrasituacional o extrasituacional) en el que estudiantes de psicología elaboraban y fundamentaban preguntas de investigación. Participaron 20 alumnos de licenciatura de una universidad pública distribuidos al azar en dos grupos: experimental y control. El grupo experimental fue expuesto a un entrenamiento correctivo en la identificación y elaboración de elementos que conforman un artículo científico. El grupo control no recibió entrenamiento (solamente leyó los mismos artículos que el grupo experimental). Los participantes fueron expuestos a una línea base, a un entrenamiento correctivo (excepto los del grupo control) y a una evaluación. Los resultados, comparados con los de un estudio previo en el que los participantes se expusieron a artículos estructurados, fueron sumamente deficientes tanto en los aciertos obtenidos como en el nivel funcional en el que formularon y fundamentaron sus preguntas de investigación, así como en la pertinencia con la que emplearon los términos técnicos al elaborarlas. Los hallazgos

María Antonia Padilla Vargas y Gonzalo Fernández Sordo, Centro de Estudios e Investigaciones en Comportamiento, Universidad de Guadalajara.

Dirigir correspondencia a los dos autores al Centro de Estudios e Investigaciones en Comportamiento, Francisco de Quevedo 180, Col. Arcos Vallarta, Guadalajara, Jalisco, México, C. P. 44130. E-mail: tony@ cencar.udg.mx, gonzalofernandezsordo@gmail.com

Se agradece a la editora de la revista, así como a los dictaminadores anónimos por sus invaluables comentarios a una versión previa de este documento, ya que éstos permitieron enriquecerlo.
\end{abstract}


son analizados en función de la importancia de identificar estrategias que permitan mejorar la forma en la que los investigadores en formación leen y escriben materiales técnicos.

Palabras clave: características textuales, preguntas de investigación, entrenamiento correctivo, artículos científicos, estudiantes universitarios.

\begin{abstract}
An experiment assessed the effects of manipulating some textual elements of scientific articles (making them non-structured) on the functional level (intra-situational or extra-situational) at which psychology undergraduate students posed and justified research questions. Twenty undergraduate students from a public university were randomly assigned to one of two groups. The experimental group was exposed to corrective training, during which participants were required to identify the elements of a scientific article and to answer questions about the article. Participants in the control group also read the articles, but did not receive corrective training. A pre-test post-test design was used. Compared to a previous study in which participants were exposed to structured articles, both the percentage of correct responses and the level at which participants elaborated and justified their research questions were considerably low. The students used the technical terms incorrectly when they stated and justified the research questions. The importance of identifying strategies to improve reading and writing of technical materials by novice researchers is discussed.

Keywords: textual characteristics, research questions, corrective training, scientific articles, undergraduate students
\end{abstract}

Al formar a un nuevo investigador el objetivo central es que sea capaz de desempeñarse de manera efectiva y novedosa, mostrando independencia intelectual respecto de quienes lo entrenaron, al plantear sus propias preguntas de investigación, lo que le permitirá consolidar sus propias líneas de generación de conocimiento (De la Fuente, Justicia, Casanova, \& Trianes, 2005; Sánchez, 2004). Ello implica que sea capaz de escribir textos técnicos en su campo disciplinar (Cassany, 2006), dado que éstos son los que permiten la generación y difusión de conocimiento (Keys, Hand, Prain, \& Collins, 1999). Debido a la naturaleza colectiva de la actividad científica, la lectura de textos técnicos es una de las actividades indispensables al realizar investigación, ya que hacer revisiones bibliográficas permite identificar lo que otros han hecho en un área de interés, y a partir de ello, plantear preguntas de investigación propias (Viniegra, 2002).

Se supone que los estudiantes de nivel medio y superior son capaces de leer comprensivamente, pero en varios estudios realizados con alumnos de psicología, comu- 
nicología y educación se ha encontrado que en realidad en tales poblaciones la comprensión lectora es inadecuada (Acuña, Irigoyen, \& Jiménez, en prensa; Arroyo, Canales, Morales, Silva, \& Carpio, 2007; Morales, Pichardo, Arroyo, Canales, Silva, \& Carpio, 2005) y la escritura es deficiente (González-Torres, Padilla-Vargas, \& Tamayo, 2011; Jiménez \& Morales, 2004; Pacheco, Ramírez, Palestina, \& Salazar, 2007; Padilla, Fuentes, \& Pacheco, en dictamen; Padilla, Solórzano, \& Pacheco, 2009; PadiIla, Suro, \& Tamayo, 2010; Padilla, Tamayo, \& González, 2010; Padilla, Tamayo, \& González-Torres, en prensa; Tamayo, Padilla, \& González-Torres, 2009; Rizo, 2004). A pesar de tal problemática, en los planes curriculares del nivel superior no se diseñan estrategias de entrenamiento en habilidades lectoescritoras (Aguilera, Alcaraz, Cabrera, Cabrera, Galicia, Hickman, et al., 2006).

Aunque se han realizado diversos trabajos, desde diferentes aproximaciones teóricas, en los que se analizaron las variables implicadas en la adquisición de la lectoescritura (Montealegre \& Forero, 2006), son pocos los que estudian qué variables influyen en el comportamiento de escribir en el nivel de pregrado y superiores en México y más escasos aún los que analizan específicamente la lectoescritura de textos técnicos, de ahí la relevancia de investigar cuáles son las variables implicadas en este fenómeno tan importante para la generación y difusión del conocimiento científico (Padilla et al., 2009).

En la perspectiva interconductual la lectoescritura se concibe como un campo de relaciones de interdependencia entre el individuo que escribe o lee y los otros elementos participantes en el episodio interactivo: las características del referente (lo que se lee o escribe), los criterios de logro, así como las características disposicionales de la situación (Pacheco, 2010).

Esta propuesta supone que escribir y leer como interacción puede estructurarse en diferentes niveles de complejidad: intrasituacionales, extrasituacionales y transituacionales (Ribes \& López, 1985). Las interacciones intrasituacionales: a) implican ajustarse a la situación respondiendo a las propiedades espacio-temporales de los eventos (e. g., al copiar, transcribir, nombrar eventos, fenómenos o cosas) o bien, b) incluyen acciones que produzcan cambios en los objetos o eventos con los que se interactúa (e. g., al escribir paráfrasis de textos previamente leídos), o c) implican que el individuo se ajuste respondiendo con precisión a condiciones cambiantes en las propiedades de los objetos o sus relaciones, para producir efectos en una situación, por ejemplo, al clasificar, con base en una tipificación o taxonomía los párrafos que componen la introducción o la discusión de un artículo leído previamente (Pacheco, 2010; Padilla et al., 2009; Ribes, Moreno, \& Padilla, 1996).

El segundo tipo, denominado extrasituacional, implica interacciones en las que un individuo responde a una situación en términos de las propiedades funcionales de otra. Este nivel es exclusivamente humano dado que requiere comportamiento lin- 
güístico, lo que posibilita reacciones desligadas de las propiedades situacionales aparentes en los eventos, objetos u organismos presentes en el ambiente. Tiene como elemento mediador a la respuesta de un sujeto con respecto a otro o consigo mismo. Por ejemplo, plantear una pregunta de investigación novedosa debidamente justificada y derivada de artículos previamente leídos. Finalmente, el tercer tipo, Ilamado transituacional involucra interacciones que implican un ajuste convencional ante objetos o eventos también convencionales. Las respuestas convencionales son las que relacionan o transforman dichos objetos o eventos. Éstos son siempre productos lingüísticos, es decir, conceptos o símbolos. Por ejemplo, elaborar una teoría como alternativa de otra existente (Ribes \& López, 1985).

En el estudio del comportamiento de leer y escribir resulta de gran importancia el análisis de los diferentes factores que participan en este tipo de interacciones, a saber: del escritor/lector, las características del referente, los criterios de dominio y ajuste que se deben cumplir al leer o escribir y las características disposicionales de la situación (Pacheco, 2010). Las características del referente comprenden aquello de lo que se escribe o se lee, por lo que resulta un factor de fundamental importancia en las interacciones. Existen dos características principales: las textuales y las funcionales. Las primeras, que serán las que se analizarán en el presente trabajo, se definen como aquellos factores relacionados con el uso apropiado de los recursos lingüísticos, como la conexión correcta de las frases de un texto, la selección de datos relevantes y la eliminación de los irrelevantes, así como la organización pertinente de los elementos de un escrito (Pacheco, 2010).

Desde una perspectiva interconductual se han tratado de identificar las variables que influyen tanto en el comportamiento lector como en el escritor. Concretamente, se han realizado estudios sobre comprensión lectora o ajuste lector que han permitido observar: 1) que los estudiantes de semestres superiores obtienen más aciertos que los de semestres iniciales en tareas que implican una interacción extrasituacional, 2) que el ajuste lector depende del tipo funcional de entrenamiento aplicado y, 3) que recibir un entrenamiento en identificación de criterios de ajuste lector favorece los desempeños en tareas tanto de identificación como de elaboración (Arroyo et al., 2005; Arroyo \& Mares, 2009; Irigoyen, Jiménez, \& Acuña, 2008; Morales, Pichardo, Arroyo, Canales, Silva, \& Carpio, 2005; Peña-Correal, Ordóñez, Fonseca, \& Fonseca, 2012). Además, se han llevado a cabo estudios que han permitido identificar la relación existente entre las habilidades de lectura y de composición escrita en estudiantes universitarios (Pacheco et al., 2007).

Concretamente, Padilla et al. (2009) realizaron un estudio en el que analizaron los efectos de entrenar a clasificar y a reformular diferentes tipos de párrafos sobre la elaboración y fundamentación de preguntas de investigación en estudiantes de posgrado. El entrenamiento promovía que los participantes identificaran algunos elementos que 
conformaban la sección introductoria de un artículo empírico (definiciones, evidencias, planteamientos derivados, objetivos, preguntas de investigación), y posteriormente, que aprendieran a reformular algunos de tales elementos, con el objeto de auspiciar que pudieran derivar preguntas de investigación novedosas, pertinentes y debidamente fundamentadas en la evidencia conceptual y empírica del área de los artículos leídos.

El entrenamiento al que se expuso a los participantes constaba de dos ejercicios diferentes: el Ejercicio 1 buscaba propiciar desempeños en un nivel intrasituacional, a partir de la clasificación de los tipos de párrafos que integran un artículo experimental dentro del análisis experimental de la conducta y la lectura de las definiciones de las variables independientes y dependientes. El Ejercicio 2 buscaba propiciar desempeños en un nivel extrasituacional, mediante la identificación de cómo los párrafos que conformaban la introducción de un artículo se vinculaban a través de la pregunta de investigación, identificando el tipo de pregunta que se planteaba así como las variables manipuladas y medidas, y finalmente completando y reformulando o parafraseando algunos párrafos del artículo previamente leído. El supuesto era que el entrenamiento en lectura comprensiva y en composición escrita de artículos empíricos, en los niveles intrasituacional y extrasituacional, promovería la elaboración y fundamentación de preguntas de investigación en un nivel extrasituacional. Los participantes debían realizar una línea base (LB) y una evaluación en la que se identificó el nivel funcional en el que elaboraban y fundamentaban una pregunta de investigación antes y después de ser expuestos al entrenamiento que constaba de los ejercicios 1 y 2 previamente descritos. La variable independiente fue la presencia/ausencia de entrenamiento (con entrenamiento, grupo experimental/ sin entrenamiento, grupo control), que consistía en identificar elementos de un artículo de investigación (Ejercicio 1) o completar, reformular o parafrasear partes de un artículo (Ejercicio 2).

Los resultados mostraron que antes del entrenamiento todos los participantes tuvieron severas dificultades para elaborar y fundamentar sus preguntas de investigación ya que ejercitaron los comportamientos lector y escritor en el nivel menos complejo (el intrasituacional), pero luego de exponerse a éste su desempeño mejoró, pues cuatro de los seis participantes lograron comportarse en niveles extrasituacionales.

En el estudio de Padilla et al. (2009) no todos los participantes del grupo experimental lograron desempeñarse en niveles extrasituacionales y ninguno de los participantes obtuvo puntajes de $100 \%$ de aciertos en las sesiones de los dos ejercicios del entrenamiento, lo cual era fundamental para que el desempeño fuera óptimo en la evaluación. Esto llevó a implementar un entrenamiento correctivo que los obligara a corregir los errores cometidos, garantizando con ello la obtención del $100 \%$ de acier- 
tos en todas las sesiones de entrenamiento. En dicho estudio se observó un hallazgo interesante. En las sesiones de entrenamiento del grupo experimental el peor desempeño de todos los participantes ocurría siempre en la segunda sesión (de tres), mientras que la ejecución era superior en las sesiones uno y tres, dato extraño puesto que se esperaría que el desempeño de los participantes fuera mejorando con el paso de las sesiones de entrenamiento.

En un análisis posterior, los autores identificaron una complejidad diferencial de los textos empleados, y por cuestiones de azar los textos más complejos fueron los empleados en las sesiones en las que se observaron las peores ejecuciones. Tal complejidad se determinó identificando la claridad con que se exponía en cada artículo la pregunta de investigación y su correspondiente fundamentación. Pero dado que llamar complejo o no complejo a un artículo puede resultar confuso se optó por denominarlo estructurado o no estructurado, a partir de los siguientes criterios: un artículo se consideraba estructurado cuando: 1) la pregunta de investigación estaba explicitada en el texto y se podían identificar claramente sus elementos (e. g., variable dependiente, variable independiente, y la vinculación entre éstas era clara), 2) se explicitaba la pertinencia de la pregunta de investigación y esos argumentos aparecían en el mismo párrafo que ésta, y 3 ) se exponía detalladamente la evidencia empírica relacionada con los diferentes elementos de la pregunta de investigación. Por otra parte, un artículo no estructurado sería aquel que no cubriera ninguno de los tres criterios enumerados.

Volpato y Gonçalves-de-Freitas (2003) sugirieron que los argumentos de un artículo científico debían ser sintéticos y suficientes para la sustentación del objetivo del trabajo y que si se omitían o no se presentaban de tal manera, el resultado era un texto difícil de leer, puesto que la sustentación del objetivo del trabajo no era clara. McNamara (2004) llevó a cabo una serie de estudios en los que manipuló agregar o eliminar información (e.g. antecedentes, referentes anafóricos y conectores) de materiales científicos utilizados en salones de clase y observó que los alumnos mejoraban su desempeño lectoescritor cuando se agregaba tal tipo de información.

Teniendo en cuenta la necesidad de identificar que los textos empleados cumplieran con los criterios de un artículo estructurado, Padilla et al. (en dictamen) Ilevaron a cabo un estudio en el que se evaluó el efecto de un entrenamiento correctivo, que garantizara la obtención del $100 \%$ de aciertos en todas las sesiones del entrenamiento en la identificación y elaboración de algunos elementos que conforman un artículo empírico, sobre el planteamiento y justificación de preguntas de investigación novedosas. Se formaron dos grupos de participantes, uno expuesto a un entrenamiento correctivo y otro sin entrenamiento correctivo, mientras que la totalidad de los materiales empleados que revisaron los participantes de ambos gru- 
pos cumplían con los tres criterios de textos estructurados. Los datos mostraron que el utilizar artículos estructurados y un entrenamiento correctivo permitió que la mayoría de los participantes del grupo experimental lograran una drástica mejora de la LB a la evaluación y que seis de los diez participantes fueran capaces de escribir y justificar sus preguntas de investigación en un nivel extrasituacional, lo que no ocurrió con los del grupo control (sin entrenamiento) en ninguno de ambos aspectos (aciertos y nivel funcional).

La posibilidad de que la manera en la que estaban estructurados los artículos a los que se expuso a los participantes pudiera haber afectado el desempeño de éstos al elaborar y fundamentar preguntas de investigación derivadas de tales textos en el estudio de Padilla et al. (2009) llevó a diseñar un nuevo estudio en el que se manipularan las características de tales textos, por lo que el objetivo del presente trabajo fue analizar los efectos de variar las características de los artículos empleados, convirtiéndolos en no estructurados, en la elaboración y fundamentación de preguntas de investigación derivadas de tales artículos, luego de exponer a los participantes a un entrenamiento correctivo en la identificación y elaboración de algunos de los elementos que conformaban dichos artículos, registrando tanto el porcentaje de aciertos obtenido como el nivel funcional en el que formulaban y fundamentaban sus preguntas de investigación.

Se emplearon los mismos textos utilizados en el estudio de Padilla et al. (en dictamen) sólo transformándolos en no estructurados, haciendo las modificaciones de acuerdo a los tres criterios previamente mencionados, usando para ello un modelo de artículo no estructurado encontrado en la literatura (que por cuestiones éticas no se menciona) y que trataba el mismo tema que el resto de los utilizados en el presente estudio. Se controló mantener las versiones modificadas lo más parecidas posible a las versiones originales en forma y extensión.

\section{Método}

\section{Participantes}

Participaron 20 alumnos de Licenciatura en psicología de una universidad pública mexicana: 14 mujeres y seis hombres de entre 20 y 22 años de edad, pertenecientes a semestres intermedios de la carrera de psicología, que participaron de manera voluntaria a cambio de créditos en uno de sus cursos académicos. Se eligió a esta población debido a que no tenían experiencia con textos del área de análisis de la conducta (elegida para el presente estudio). Dado que los sujetos provenían de una misma escuela tenían un conocimiento uniforme (mínimo) en lo relativo a me- 
todología de la investigación (e. g., identificación de variables independientes y dependientes).

\section{Materiales}

Se utilizaron cuatro artículos experimentales en los que se analizaban los efectos de la retroalimentación y las instrucciones en tareas de discriminación condicional. Todos los estudios fueron llevados a cabo en el marco del análisis de la conducta y tenían una extensión de entre 10 a 14 cuartillas. Además se usaron diversos materiales diseñados ex profeso, los cuales se describen en el apartado de Procedimiento. Se eligieron tales artículos en concreto debido a que pertenecían a una misma área de investigación, tenían una extensión similar, pero sobre todo, debido a que cumplían con los requisitos de: 1) tener claramente descrita la pregunta de investigación con su variable independiente, dependiente, y donde fuera clara la vinculación entre éstas, 2) que se argumentara la pertinencia de la pregunta de investigación y dichos argumentos aparecieran en el mismo párrafo que la pregunta, y 3) que se expusiera detaIladamente la evidencia empírica relacionada con los diferentes elementos de la pregunta de investigación correspondiente. Dado que cumplían los tres criterios previos se consideraron artículos estructurados, pero se convirtieron en no estructurados para utilizarlos en el presente estudio, al evitar que tuvieran tales características (ver en el Apéndice el listado de los artículos empleados).

\section{Diseño}

Se empleó un diseño intergrupo. Los participantes se asignaron de manera aleatoria a dos grupos: experimental y control. El grupo experimental fue expuesto a los artículos no estructurados y a entrenamiento correctivo mientras que el control sólo leyó los artículos no estructurados pero no recibió entrenamiento. El entrenamiento correctivo consistió en exponer a los alumnos a la tarea, inmediatamente después de realizada se les indicaron los errores cometidos, y ellos los debían corregir. Posteriormente se volvía a revisar lo realizado, a la tercera ocasión en que se siguiera cometiendo algún error después de las correcciones se les decía la respuesta correcta y se continuaba con la siguiente condición experimental. Ambos grupos se expusieron a una LB, al entrenamiento (excepto el control) y a la evaluación. En la Tabla 1 se muestra el diseño experimental empleado. Los artículos estructurados eran textos que cumplían con los tres criterios descritos en la sección de Materiales mientras que los no estructurados eran los mismos trabajos ya mencionados pero a los que se les hicieron modificaciones en las secciones correspondientes, para que no cumplieran con ninguno de tales criterios. 
Tabla 1

Diseño experimental empleado

\begin{tabular}{|c|c|c|c|c|}
\hline Grupos & & & ses & \\
\hline Experimental & Línea base & Ejercicio 1 & Ejercicio 2 & Evaluación \\
\hline Control & Línea base & Sólo lectura & Sólo lectura & Evaluación \\
\hline
\end{tabular}

\section{Escenario}

Las sesiones experimentales se llevaron a cabo en cubículos de dos metros cuadrados, sonoamortiguados y que contaban con iluminación natural y artificial. En cada cubículo había una mesa, una silla y un equipo de cómputo.

\section{Procedimiento}

Los participantes resolvían la tarea individualmente. Antes de iniciar la tarea leían, y en su caso firmaban, un formato de consentimiento informado. Los participantes del grupo experimental se exponían a siete sesiones y los del grupo control a cuatro. Las sesiones tenían una duración aproximada de una hora, y se realizaba una sesión por día. Ambos grupos debían leer dos artículos y elaborar una pregunta y su correspondiente fundamentación tanto en la fase de LB como en la de evaluación. Los del grupo experimental se exponían a dos sesiones de $L B$, en la primera de éstas debían leer un texto y si lo deseaban, podían hacer anotaciones en una hoja. En la segunda sesión de LB debían leer un segundo texto y contestar algunas preguntas respecto de la pregunta de investigación del artículo leído, el tipo de pregunta de éste (con base en una clasificación diseñada ex profeso) y mencionar las variables independientes y las variables dependientes del estudio. Finalmente, con base en los dos artículos leídos y una guía diseñada para tal fin, debían elaborar un anteproyecto de investigación que contuviera: una pregunta de investigación, por lo menos una variable dependiente, una variable independiente, evidencia(s) que diera(n) soporte a la pregunta elaborada, argumentos que justificaran la propuesta, describir qué participantes sería conveniente emplear para responder a la pregunta elaborada, el procedimiento, los resultados esperados y explicar de qué manera creían que se vinculaban tales resultados con la pregunta de investigación propuesta.

En una segunda fase se exponía al grupo experimental a un entrenamiento que constaba de dos sesiones para cada uno de dos ejercicios. El Ejercicio 1 (diseñado para promover desempeños en niveles intrasituacionales) consistía en leer un artículo e identificar ciertos elementos que componían la introducción, leer un texto acer- 
ca de las preguntas de investigación (preparado ex profeso) y responder a las mismas preguntas que en la segunda sesión de LB. Además debían identificar en qué parte del artículo había: Definiciones, Evidencias, Formulación de preguntas y Objetivos, Planteamientos derivados o una combinación de dos o más de tales elementos. Los participantes se exponían a dos sesiones con dos artículos diferentes y al concluir cada sesión se retroalimentaba su desempeño, y en cada caso debían corregir sus errores. Tenían tres oportunidades para obtener la respuesta correcta, y si no lo hacían en la tercera ocasión se les indicaban éstas y se continuaba con la siguiente sesión.

El Ejercicio 2 (diseñado para auspiciar desempeños en niveles extrasituacionales) también constaba de dos sesiones, en cada una de las cuales el participante debía leer los mismos artículos que en el ejercicio anterior y a partir de la lectura de cada uno de tales textos debía responder a las preguntas: ¿Cómo se relacionaba la introducción con la pregunta de investigación del artículo leído?, ¿Cómo se justificaba la elaboración del estudio?, ¿Cómo se vinculaban las evidencias presentadas en la introducción con los resultados? Además, debía proponer una tarea diferente a la empleada en el artículo leído para medir las mismas variables analizadas en éste y proponer el estudio de una variable no contemplada en tal artículo y que fuera pertinente al área analizada en dicho texto. Por otra parte, debía formular una pregunta de investigación que incluyera la variable propuesta, explicitando variables dependientes e independientes. Finalmente debía explicar resultados alternativos con base en lo expuesto por el autor del artículo leído. La retroalimentación se proporcionaba de forma similar al Ejercicio 1.

Se consideraba que este ejercicio promovía desempeños en niveles extrasituacionales dado que implicaba que los participantes no repitieran lo leído, sino que a partir de los artículos revisados identificaran que lo que articula y le da coherencia a un artículo empírico es la pregunta de investigación, por lo que debían analizarlos de manera crítica, elaborando, formulando, fundamentando, e interpretando evidencia, para finalmente poder elaborar su pregunta de investigación derivada de éstos.

Por otra parte, la fase de evaluación se llevaba a cabo en una sola sesión e implicaba elaborar una pregunta de investigación y su correspondiente justificación basándose en los textos leídos anteriormente y con la misma guía empleada en la LB. Al concluir se les proporcionaba retroalimentación respecto de su ejecución. Los participantes del grupo control leían los mismos artículos que el experimental para la LB y la Evaluación, pero no se les proporcionaba ningún tipo de entrenamiento ni retroalimentación.

\section{Registro de los datos}

El análisis de los datos recabados se realizó tanto cuantitativa como cualitativamente. Para la parte cuantitativa se registró el porcentaje de respuestas correctas obtenido 
por cada participante en la LB, los ejercicios 1 y 2 y en la evaluación empleando para ello un Baremo diseñado ex profeso con el que se calificaba el desempeño en cada aspecto, por ejemplo, en el caso específico de las evidencias que dieran soporte a la pregunta de investigación propuesta, se asignaban dos puntos si se mencionaban por lo menos dos evidencias distintas, se explicaba su pertinencia y si éstas eran adecuadas (en las instrucciones se clarificaba que se debían mencionar por lo menos dos evidencias), se asignaba un punto si solo se mencionaba una evidencia pertinente ó se mencionaban dos, pero no se explicaba su pertinencia, y cero puntos si no mencionaban evidencias o las que describían no eran pertinentes. Y lo mismo se hacía para cada uno de los siguientes aspectos: elaboración de la pregunta de investigación, identificación de las variables independiente y dependiente, argumentación para justificar la propuesta, descripción de participantes a emplear en el estudio propuesto, información o datos a recabar en el estudio, predicción de resultados y vinculación de resultados esperados con la pregunta de investigación propuesta.

Con respecto al aspecto cualitativo, se identificaba el nivel funcional en el que se elaboraba y justificaba la pregunta de investigación en la LB y en la evaluación, analizando si éste mejoraba de una condición a otra como efecto del entrenamiento. Para el análisis del nivel funcional en el que se planteaba y fundamentaba la pregunta de investigación se emplearon los siguientes criterios: una pregunta y su correspondiente justificación se consideraban escritas en un nivel Intrasituacional cuando ésta o su justificación eran meras copias de los artículos previamente leídos o cuando no había correspondencia entre la pregunta y la justificación propuestas. Se consideraban Extrasituacionales si se proponía una manipulación que no se hubiera realizado en los artículos revisados o se modificaba el diseño, método o análisis propuestos; si ésta se derivaba lógicamente de lo leído, y si había congruencia entre la pregunta de investigación y su justificación. Dado que el criterio de esta tarea era que se elaborara una pregunta derivada, cuando la propuesta del participante no tenía ninguna relación con los artículos leídos ésta se consideraba no pertinente y se evaluaba como ausencia de respuesta. Con el objeto de dar confiabilidad al análisis de resultados las respuestas de los participantes fueron analizadas por dos calificadores independientes. En caso de discrepancia se discutía cada dato hasta llegar a un acuerdo, por lo que la confiabilidad fue de $100 \%$ en todos los casos.

\section{Resultados}

La Figura 1 muestra que la mayoría de los participantes del grupo experimental aumentó mínimamente su puntaje de la LB a la evaluación e incluso con ello jamás superaron el $65 \%$ de aciertos, mientras que ninguno del grupo control obtuvo más del $60 \%$. En la Tabla 2 puede observarse que únicamente dos de los diez participan- 
tes del grupo experimental (P04 y P09) escribieron sus preguntas de investigación en un nivel extrasituacional en la evaluación mientras que ninguno de los sujetos del grupo control las elaboró en este nivel en dicha condición.

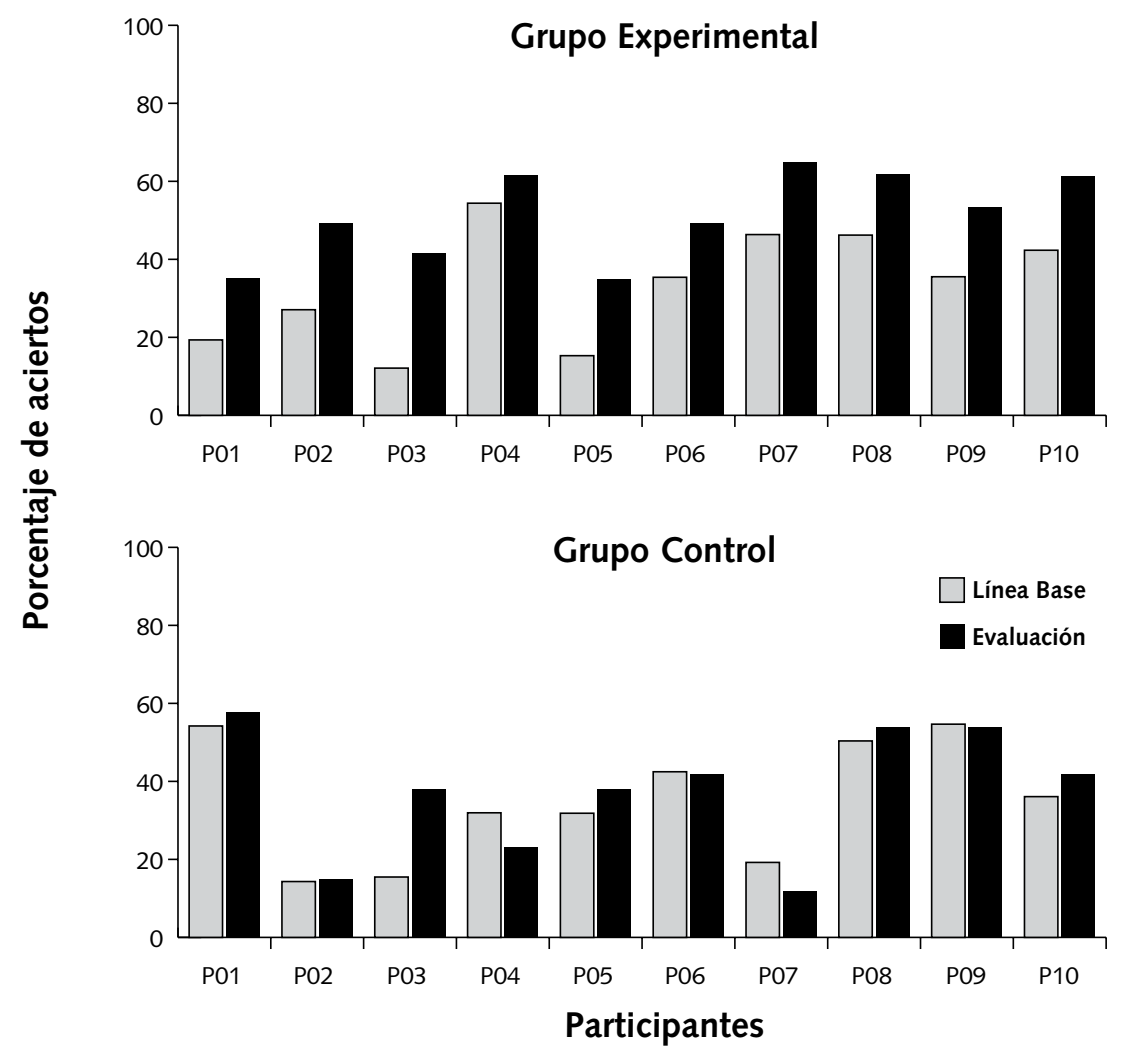

Figura 1. Porcentaje de respuestas correctas obtenido por los participantes de los grupos Experimental y Control en las condiciones de Línea Base y Evaluación.

En la Figura 2 se muestra el porcentaje de aciertos obtenido en el entrenamiento. Prácticamente todos obtuvieron un puntaje por debajo del $60 \%$ de respuestas correctas en ambos ejercicios (con excepción de los participantes P09 y P10 en el Ejercicio 1, y P04, P07, P09 y P10, en el Ejercicio 2). Todos los hallazgos previamente descritos parecen indicar que exponerse a un entrenamiento correctivo con artículos no estructurados no mejora sustancialmente el desempeño, dado que aunque los participantes del grupo experimental sí aumentaron su puntaje de la LB a la evaluación nunca superaron el $65 \%$ de aciertos. 
Tabla 2

Nivel funcional en que fueron escritas las preguntas de investigación

Grupo Experimental

\begin{tabular}{|c|c|c|c|}
\hline Participante & Condición & $\begin{array}{l}\text { Nivel } \\
\text { Funcional }\end{array}$ & $\begin{array}{l}\text { Tiempo } \\
\text { en minutos }\end{array}$ \\
\hline \multirow[t]{2}{*}{ P01 } & LB & I & 62 \\
\hline & $\mathrm{Ev}$ & I & 98 \\
\hline \multirow[t]{2}{*}{ P02 } & LB & 1 & 62 \\
\hline & Ev & I & 62 \\
\hline \multirow[t]{2}{*}{ P03 } & LB & $x$ & 71 \\
\hline & $\mathrm{Ev}$ & I & 110 \\
\hline \multirow[t]{2}{*}{ P04 } & LB & I & 108 \\
\hline & $\mathrm{Ev}$ & $\mathrm{E}$ & 104 \\
\hline \multirow[t]{2}{*}{ P05 } & LB & $x$ & 106 \\
\hline & $\mathrm{Ev}$ & $x$ & 60 \\
\hline \multirow[t]{2}{*}{ P06 } & LB & I & 103 \\
\hline & Ev & I & 69 \\
\hline \multirow[t]{2}{*}{ P07 } & LB & I & 70 \\
\hline & Ev & I & 72 \\
\hline \multirow[t]{2}{*}{ P08 } & LB & I & 50 \\
\hline & Ev & I & 70 \\
\hline \multirow[t]{2}{*}{ P09 } & LB & I & 71 \\
\hline & Ev & $E$ & 62 \\
\hline \multirow[t]{2}{*}{ P10 } & LB & I & 84 \\
\hline & Ev & I & 96 \\
\hline
\end{tabular}

Nota: $\left(E=\right.$ Extrasituacional; $I=$ Intrasituacional; $X=$ Ausencia de respuesta ${ }^{*}$ y tiempo invertido para elaborarlas en la Línea base y en la Evaluación.

* Cuando la pregunta elaborada no tenía relación con los artículos leídos se consideraba como no pertinente y se evaluaba como ausencia de respuesta. 
Tabla 2 (continuación)

Nivel funcional en que fueron escritas las preguntas de investigación

Grupo Control

\begin{tabular}{|c|c|c|c|}
\hline Participante & Condición & $\begin{array}{l}\text { Nivel } \\
\text { Funcional }\end{array}$ & $\begin{array}{l}\text { Tiempo } \\
\text { en minutos }\end{array}$ \\
\hline \multirow[t]{2}{*}{ P01 } & LB & 1 & 62 \\
\hline & Ev & I & 79 \\
\hline \multirow[t]{2}{*}{ P02 } & LB & 1 & 67 \\
\hline & Ev & I & 53 \\
\hline \multirow[t]{2}{*}{ P03 } & LB & I & 49 \\
\hline & Ev & I & 70 \\
\hline \multirow[t]{2}{*}{ P04 } & LB & I & 80 \\
\hline & Ev & I & 84 \\
\hline \multirow[t]{2}{*}{ P05 } & LB & I & 76 \\
\hline & Ev & I & 84 \\
\hline \multirow[t]{2}{*}{ P06 } & LB & I & 83 \\
\hline & Ev & I & 76 \\
\hline \multirow[t]{2}{*}{ P07 } & LB & $x$ & 66 \\
\hline & Ev & $x$ & 88 \\
\hline \multirow[t]{2}{*}{ P08 } & LB & I & 81 \\
\hline & Ev & I & 87 \\
\hline \multirow[t]{2}{*}{ P09 } & LB & $E$ & 154 \\
\hline & Ev & I & 112 \\
\hline \multirow[t]{2}{*}{ P10 } & LB & I & 88 \\
\hline & Ev & I & 84 \\
\hline
\end{tabular}

Nota: $(E=$ Extrasituacional; $I=$ Intrasituacional; $X=$ Ausencia de respuesta $*$ y tiempo invertido para elaborarlas en la Línea Base y en la Evaluación.

* Cuando la pregunta elaborada no tenía relación con los artículos leídos se consideraba como no pertinente y se evaluaba como ausencia de respuesta. 


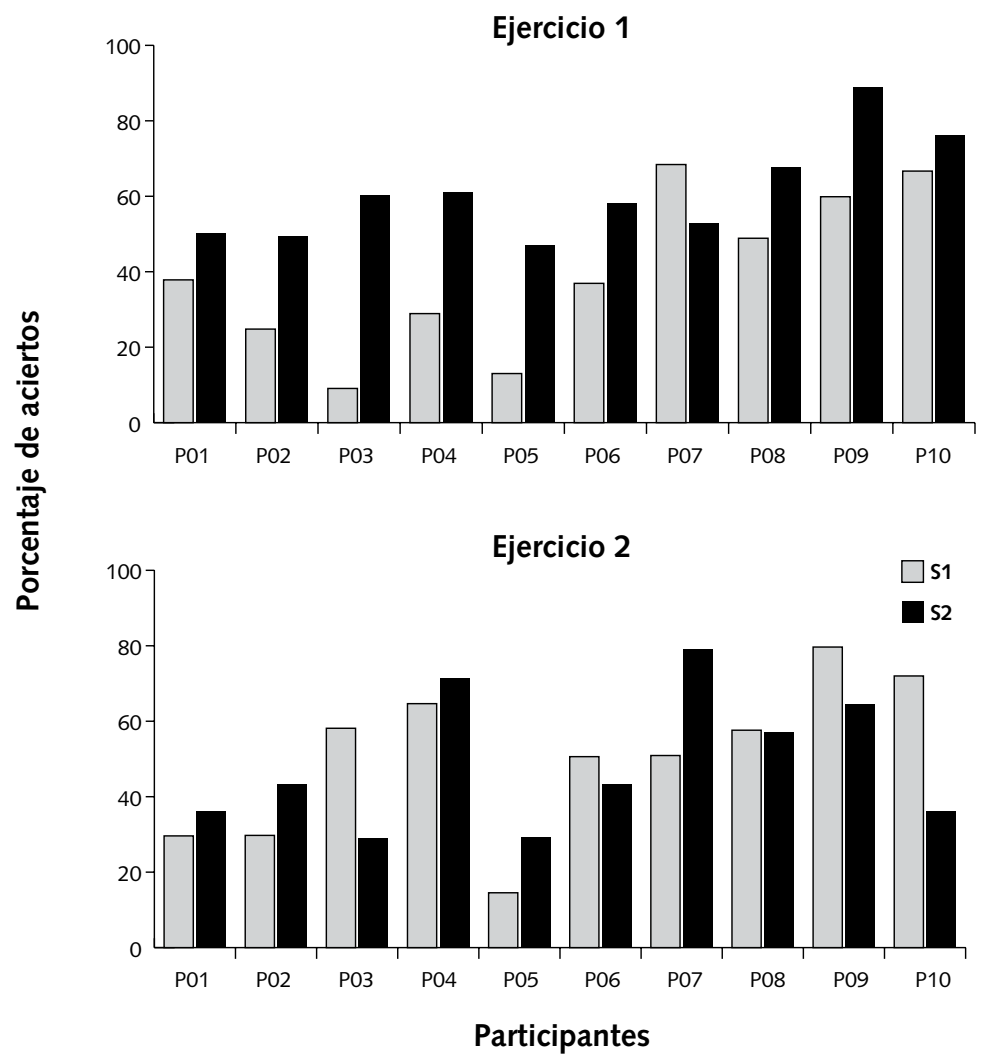

Figura 2. Porcentaje de aciertos obtenido por los participantes del grupo Experimental en las dos sesiones de cada uno de los ejercicios de entrenamiento. Claves: S1 = Sesión 1; S2 = Sesión 2.

Con respecto al número de palabras empleado por los participantes del grupo experimental en la elaboración y fundamentación de sus preguntas de investigación, éstos aumentaron la extensión de la LB a la evaluación en promedio en 276 palabras, pero sólo cuatro de los diez participantes (P02, P04, P08 y P09) utilizaron los términos técnicos de forma pertinente en la fase de evaluación pero no en la LB. Con respecto al desempeño del grupo control, se observó que la mitad disminuyó el número de palabras de su escrito mientras que la otra mitad lo aumentó pero ello no pareció guardar una relación con el porcentaje de aciertos obtenido.

Para enfatizar las diferencias entre exponerse a textos estructurados o no estructurados en la Figura 3 se muestra una comparación del promedio del porcentaje de aciertos de los participantes del grupo experimental del estudio de Padilla et al. (en 

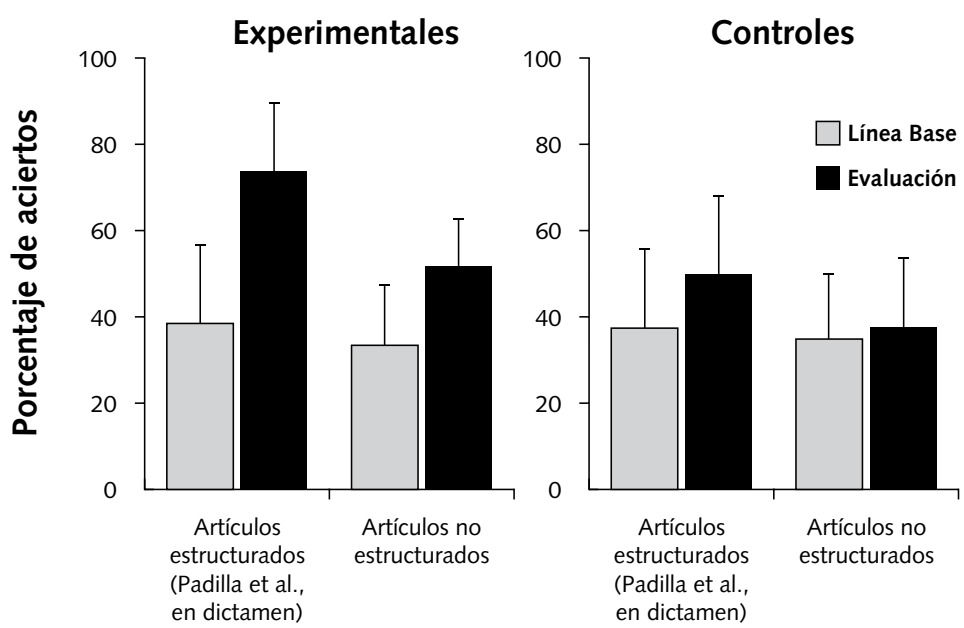

Figura 3. Porcentaje promedio de aciertos (con desviación estándar) obtenido por los participantes de los grupos Experimental y Control del estudio de Padilla, Fuentes y Pacheco (en dictamen) expuestos a artículos estructurados y de los grupos Experimental y Control del presente estudio, expuestos a artículos no estructurados, en las fases de Línea Base y Evaluación.

dictamen), y del presente estudio en las fases de LB y evaluación. Puede observarse que, en promedio, los puntajes en LB de los participantes de los grupos expuestos a artículos estructurados o no estructurados fueron similares $(M=38$ y 33 , respectivamente, $D E=18.61$ y 14.39), pero en la evaluación el grupo expuesto a artículos estructurados obtuvo una media de $74(D E=15.66)$, lo que implica un aumento promedio de $36 \%$ de aciertos de la LB a la evaluación, mientras que el que había sido entrenado con artículos no estructurados obtuvo en promedio un porcentaje de aciertos de $51 \%$ en la evaluación $(D E=11.34)$, lo que significa que aumentó sólo $18 \%$ de una condición a otra (ver Figura 3). Es importante mencionar que si bien en el estudio de Padilla et al. (en dictamen) la mayoría de los participantes obtuvo puntajes entre $80 \%$ y $100 \%$ de aciertos, hubieron dos con desempeños extremadamente bajos que afectaron el promedio general.

En cuanto al desempeño del grupo control del estudio de Padilla et al. (en dictamen) y del presente estudio, en la LB sus puntajes fueron similares independientemente del tipo de artículos al que se expusieron: estructurados o no estructurados (37 y $34 \%$, respectivamente). Mientras que en la evaluación el grupo que se enfrentó a artículos estructurados obtuvo un puntaje de $50 \%$, lo que implica un aumento promedio de $13 \%$ de aciertos, de la LB a la evaluación; en tanto que el expuesto a artículos no estructurados obtuvo en promedio un porcentaje de aciertos de $37 \%$ en la evaluación, es decir, que no aumentó su puntaje de la LB a la evaluación. 


\section{Discusión}

El objetivo del presente trabajo fue analizar los efectos de exponerse a un entrenamiento correctivo, empleando textos no estructurados, al elaborar y fundamentar preguntas de investigación. Los resultados mostraron que el grupo experimental (con entrenamiento correctivo) tuvo una mejora mínima en su desempeño de la LB a la evaluación, al contrario del grupo control cuyos puntajes fueron similares de una condición a la otra. Mientras que en el estudio de Padilla et al. (en dictamen) se había observado que la gran mayoría de los participantes (con y sin entrenamiento) que revisaron artículos estructurados habían logrado un aumento sustancial en el número de aciertos de la LB a la evaluación.

Como puede observarse en la Tabla 2, si bien el tiempo que le llevó a cada participante realizar las actividades requeridas en las condiciones de LB y evaluación fue diferente para cada uno, ello no parece haberse relacionado con el desempeño, ya que como se muestra en la Figura 1, algunos de los sujetos que invirtieron más tiempo en la LB, por ejemplo, tuvieron uno de los desempeños más bajos y a la inversa (ver P05 y 08). Adicionalmente, el que los participantes del grupo control no hayan mejorado su desempeño a pesar de que prácticamente duplicaron el tiempo invertido en la realización de las actividades de la evaluación con respecto a la $L B$, mientras que los del grupo experimental sí mejoraron con cambios mínimos en sus tiempos, parece ser un indicador de que el efecto observado se debió al tratamiento experimental al que se les expuso, y no al tiempo que les llevó concluir las tareas requeridas.

En lo que respecta a los porcentajes de aciertos obtenidos en los ejercicios de identificación y elaboración, se encontró que éstos fueron bajos, a pesar del entrenamiento correctivo, a diferencia de exponerse a entrenamiento con artículos estructurados. Los resultados del presente estudio son congruentes con el hallazgo de Padilla et al. (2009) quienes habían observado que cuando los participantes se habían entrenado con artículos posteriormente calificados como no estructurados su desempeño había sido deficiente. Al respecto puede concluirse que cuando un lector no entrenado, es decir, sin formación como investigador, o con poca experiencia en la lectoescritura de artículos experimentales, se expone a un texto en el que la pregunta de investigación no está explicita o es poco clara, en el sentido que no se identifica alguno de sus elementos o no se aclara la vinculación entre las variables implicadas, además de que no se explicita su pertinencia o no se expone de manera ordenada la evidencia que justifica dicha pregunta, ello dificulta el desempeño tanto en los ejercicios de identificación como en los de elaboración, según parecen indicar los haIlazgos tanto del estudio de Padilla et al. (2009) como los del presente trabajo.

La exposición a textos no estructurados parece que no sólo afecta la ejecución en términos del porcentaje de aciertos, sino también en lo relativo al nivel funcional en 
el que se escribe y fundamenta una pregunta de investigación. Padilla et al. (en dictamen), encontraron una relación entre el porcentaje de respuestas correctas logradas en el ejercicio de elaboración y el nivel en que los participantes elaboraban y justificaban sus preguntas de investigación, ya que observaron que un bajo porcentaje de aciertos se relacionaba con un desempeño en niveles intrasituacionales y un alto puntaje con una ejecución en niveles extrasituacionales. Este efecto ha sido previamente reportado por otros autores, quienes han encontrado que el nivel funcional en el que se estructura una competencia verbal modula la probabilidad de que ese individuo se comporte en ese mismo nivel cuando se expone a situaciones novedosas (Mares, Ribes, \& Rueda, 1993; Mares, Rueda, Plancarte, \& Guevara, 1997).

En el estudio de Padilla et al. (en dictamen), se había encontrado que el entrenamiento correctivo lograba promover la elaboración y justificación de preguntas de investigación en niveles extrasituacionales pero lo que se observó en el presente trabajo fue que exponerse a dicho entrenamiento correctivo, empleando para ello artículos no estructurados, no tuvo efectos en dicho sentido ya que la elaboración y fundamentación de preguntas de investigación en un nivel extrasituacional sólo se observó en dos de los diez participantes, en la evaluación. Estos hallazgos podrían explicarse en función de lo que señalaron varios autores (Cassany, 2006; De la Fuente et al., 2005; Keys et al., 1999; Sánchez, 2004), respecto de la importancia de derivar las nuevas preguntas de investigación de un contexto teórico y empírico específico, lo que implica por una parte, ser capaz de leer materiales técnicos (e. g., Ejercicio 1) y por otra parte, escribir en niveles extrasituacionales (e. g., Ejercicio 2), dado que elaborar preguntas novedosas y pertinentes derivadas de los artículos previamente leídos implicaba, por lo menos, analizar críticamente lo propuesto por el autor de cada artículo revisado y no sólo repetir lo ya realizado o leído, lo que demandaría sólo comportarse en un nivel intrasituacional, que fue lo que se observó en el presente estudio. Al parecer, exponer a los participantes a artículos no estructurados promueve un desempeño deficiente tanto en el puntaje de aciertos logrado como en el nivel funcional en el que se elaboran y fundamentan preguntas de investigación, en comparación con exponerse a artículos estructurados.

Para poder afirmar que un comportamiento ha tenido lugar en un nivel extrasituacional se deben cumplir los siguientes criterios: 1 ) ocurrir a través de un sistema convencional, 2) que existan dos momentos de respuesta, 3) se dé un desligamiento funcional de las propiedades situacionales de los eventos con los que se entra en contacto, y 4) se den relaciones de condicionalidad que no dependan de las propiedades fisicoquímicas y biológicas de los eventos y elementos de respuesta involucrados (Ribes \& López, 1985). Se considera que el desempeño de algunos de los participantes ocurrió en un nivel extrasituacional dado que la tarea implicaba lectoescritura (sistema convencional), había dos momentos de respuesta (LB y evaluación), 
la tarea implicaba desligarse de la situación ya que se debía elaborar una pregunta novedosa y las relaciones de condicionalidad que tenían lugar en los casos en que se actualizó dicha función no dependían de las propiedades fisicoquímicas y biológicas de los materiales empleados (se entraba en contacto con las propiedades lingüísticas de los textos).

El bajo incremento logrado en el porcentaje de respuestas correctas de la LB a la evaluación en los participantes del grupo experimental parece demostrar el efecto disminuido del entrenamiento correctivo cuando para ello se emplean artículos no estructurados. Es importante destacar que la mayoría de los errores observados se relacionaron con una elaboración incompleta o poco clara de la pregunta de investigación y la ausencia de argumentos claros en su fundamentación. Aunque el entrenamiento correctivo tenía como objetivo entrenar a los participantes a identificar las variables dependientes e independientes; identificar la relación entre éstas y el cómo se articula una pregunta de investigación a partir de la evidencia que se presenta en la introducción de un artículo, los datos demostraron que exponerse a artículos no estructurados interfirió con dicho logro, al contrario de lo que ocurrió al enfrentarse a textos estructurados (Padilla et al., en dictamen). Ello parece implicar que modificar las propiedades o características del referente, en este caso particular, darle una estructura ligeramente caótica a un artículo, crear ambigüedad y eliminar algunos elementos en éste, dificultó el desempeño de los participantes, tanto en el entrenamiento como en la elaboración y fundamentación de sus preguntas. Es decir, la situación de entrenamiento a la que fueron expuestos los participantes no promovió condiciones propicias para el desarrollo de habilidades vinculadas con la lectura (e. g., identificación de elementos relevantes de un texto) y la composición escrita de textos científicos.

Según Johnstone, Ashbaugh y Warfield (2002) la exposición repetida a los términos técnicos de una disciplina (contaduría, negocios y mercadotecnia) mejoran las habilidades escritoras de los alumnos. Lo mismo mostraron Kelly y Chen (1999), quienes encontraron que estudiantes de secundaria escribían de manera más sistemática y utilizaban un mayor número de términos científicos correspondientes a la teoría entrenada después de ser expuestos a un procedimiento en el que se les explicitaba claramente el objetivo a lograr con esa actividad y se les proporcionaba una guía que tenía como meta enseñarles a escribir de manera ordenada. Si ello se logró con estudiantes de secundaria, con grados superiores el proceso debería resultar más sencillo, como ya se ha demostrado cuando para tal meta se emplea ejercicio supervisado, modelamiento, evaluación-corrección y explicitación de los criterios pedagógicos y disciplinares a cumplir (Pacheco, 2010; Padilla et al., 2009; Padilla et al., en dictamen).

Cabe destacar que en el presente estudio se controló lo relativo a la historia de referencialidad de los participantes con el tipo de textos a los que se les expuso, es 
decir, la familiaridad que se tiene con lo que se debe leer o escribir (Pacheco, 2010). Se verificó que los participantes no hubieran tenido contacto previo con el tipo de referente al que se enfrentaron, ya que provenían de una licenciatura en la que no existen laboratorios experimentales y no se revisan textos del análisis experimental de la conducta. De esta manera se garantizó que los resultados obtenidos fueran efecto de la preparación experimental empleada y no de la experiencia diferencial de los participantes con la temática de los textos que debían leer.

Se considera importante controlar la historia de referencialidad para que no se convierta en una posible variable extraña, si lo que se pretende es analizar el efecto de exponer a los participantes a artículos estructurados o no estructurados, ya que en un estudio realizado por Padilla y González (2013), quienes expusieron a investigadores experimentados al mismo diseño empleado en el presente trabajo, es decir, utilizando los mismos artículos estructurados y no estructurados, los resultados mostraron que la experiencia de los investigadores contrarrestó el efecto de la estructura de los artículos, ya que desde la LB el desempeño de los participantes fue alto (cercano al $60 \%$, independientemente del tipo de artículos al que hubieran sido expuestos -estructurados o no estructurados-) y en la evaluación el desempeño de ambos grupos fue cercano al $100 \%$ de aciertos, a diferencia de lo observado en este estudio, en el que sí se controló la historia de referencialidad de los sujetos.

El hecho de que los participantes del presente estudio hayan aumentado la extensión de la elaboración y fundamentación de sus preguntas de investigación de la LB a la evaluación concuerda con hallazgos previos (Padilla et al., 2009; Padilla et al., en dictamen), lo cual era esperable dado que en la evaluación los participantes ya habían revisado cuatro artículos del área, y no sólo dos, como era el caso de la LB, lo que les permitía contar con mayores elementos para justificar y argumentar su propuesta, aunque el aumento fue menor al observado en trabajos previos. Pero lo diferente fue la pertinencia con la que usaron tales argumentos, dado que en estudios previos se había observado que en la evaluación, además de aumentar sustancialmente la extensión de sus propuestas, la justificación de las preguntas de investigación se hacía utilizando los términos técnicos del área de manera pertinente, lo que ocurrió en el presente estudio sólo en cuatro de los diez participantes, y ello sólo en el grupo experimental. Los resultados del presente estudio pueden explicarse en función de los hallazgos de Padilla et al. (2010) quienes realizaron un experimento con el objetivo de identificar los efectos del domino diferencial de los supuestos teóricos y metodológicos de una teoría sobre la elaboración y justificación de preguntas de investigación. En dicho trabajo expusieron a estudiantes de licenciatura a un entrenamiento diferencial en los supuestos de la teoría piagetana y encontraron que a mayor exposición a los elementos de dicha perspectiva (definiciones, ejemplos y experimentos), mayor fue la cantidad de participantes que emplearon términos técnicos de ésta 
para justificar su pregunta de investigación, además de que a mayor exposición a tales postulados, los participantes elaboraban y fundamentaban preguntas de investigación de manera más apegada a la lógica de dicha teoría. Por ello se considera que el hallazgo de un aumento en el uso de términos técnicos de manera pertinente de la LB a la evaluación, pero sólo por unos cuantos participantes, observado en el presente estudio, es consistente con los hallazgos de Padilla et al. (2010), dado que si bien en este estudio los sujetos se exponían a cuatro artículos experimentales, el hecho de que se hubiera modificado su estructura al parecer les dificultó identificar y usar los términos técnicos de manera pertinente, lo que funcionalmente parece ser equivalente a exponerse sólo a algunos elementos de una propuesta teórica.

Dado lo anteriormente señalado, se considera que el estudio sistemático de las características textuales de materiales técnicos podría permitir la elaboración de un entrenamiento efectivo de comprensión lectora y composición escrita que podría ser empleado como estrategia didáctica en tareas que impliquen leer y escribir textos técnicos.

Finalmente, debido que hasta ahora los análisis realizados en el área se han limitado a evaluar el efecto de las características textuales del referente, faltarían por estudiarse las variables relativas a las características funcionales de los textos técnicos. Por otra parte, se considera necesario hacer un análisis sistemático de cada uno de los elementos implicados en un episodio interactivo de lectoescritura, como son los relativos al que escribe o lee, los del referente, los criterios de dominio y ajuste que se deben cumplir al leer o escribir (Arroyo et al., 2007), y las características disposicionales de la situación (Pacheco, 2010).

\section{Referencias}

Acuña, K. F., Irigoyen, J. J., \& Jiménez, M. Y. (en prensa). La comprensión de contenidos científicos en estudiantes universitarios. México: UniSon-Qartuppi.

Aguilera, G., Alcaraz, R., Cabrera, R., Cabrera, N., Galicia, X., Hickman, H... Robles, S. (2006). Comisión de Evaluación del Plan de Estudios Vigente (Documento interno). México: FES-Iztacala.

Arroyo, R., Canales, C., Morales, G., Silva, H., \& Carpio, C. (2007). Programa de investigación para el análisis funcional del ajuste lector. Acta Colombiana de Psicología, 10 (2), 31-39.

Arroyo, R., \& Mares, G. (2009). Efectos del tipo funcional de entrenamiento sobre el ajuste lector. Revista Mexicana de Análisis de la Conducta, 35(1), 19-35.

Cassany, D. (2006). Taller de textos. Leer, escribir y comentar en el aula. España: Paidós.

De la Fuente, J., Justicia, F., Casanova, P. F., \& Trianes, M. V. (2005). Perceptions about the construction of academic and professional competencies in psychologists. 
Electronic Journal of Research in Educational Psychology, 3 (1), 3-34.

González-Torres, M. L., Padilla-Vargas, M. A., \& Tamayo, J. (2011). Effects of the degree of specificity of the achievement criterion on the informal questions elaboration. International Journal of Hispanic Psychology, 3 (2), 171-183.

Irigoyen, J., Jiménez, M., \& Acuña, K. (2008). Análisis de la competencia lectora en estudiantes universitarios. Boletín electrónico de la Asociación Oaxaqueña de Psicología, 4(1), 84-96.

Jiménez, S.A., \& Morales, J. (2004). La capacidad para la investigación en las trayectorias escolares de maestría. En: Secretaría de Educación Jalisco (Comp.) Antología de seminarios de investigación: formación docente. 2004. Recuperado el 12 de abril de 2008, de http://educacion.jalisco.gob.mx

Johnstone, K. M., Ashbaugh, H., \& Warfield, T. D. (2002). Effects of repeated practice and contextual-writing experiences on college students' writing skills. Journal of Educational Psychology, 94 (2), 305.

Kelly, G., \& Chen, C. (1999). The sound of music: constructing science as sociocultural practices through oral and writing discourse. Journal of Research in Science Teaching, 36 (8), 883-915.

Keys, C. W., Hand, B., Prain, V., \& Collins, S. (1999). Using the science writing heuristic as a tool for learning from laboratory investigations in secondary science. Journal of Research in Science Teaching, 36 (10), 1065-1084.

Mares, G., Ribes, E., \& Rueda, E. (1993). El nivel de funcionalidad en lectura y su efecto sobre la transferencia de lo leído. Revista Sonorense de Psicología, 7 (1), 32-43.

Mares, G., Rueda P, E., Plancarte, P., \& Guevara, Y. (1997). Conducta referencial no entrenada: el papel que juega el nivel funcional de entrenamiento en la generalización. Acta Comportamentalia, 5 (2), 199-219.

McNamara, D. S. (2004). Aprender del texto: Efectos de la estructura textual y las estrategias del lector. Revista Signos, 37 (55), 19-30.

Montealegre, R., \& Forero, L. A. (2006). Desarrollo de la lectoescritura: Adquisición y dominio. Acta Colombiana de Psicología, 9 (1), 25-40.

Morales, G., Canales, C., Arroyo, R., Pichardo, A., Silva, H., \& Carpio, C. (2005). Efectos del entrenamiento en la identificación de criterios de ajuste lector en estudiantes universitarios. Enseñanza e Investigación en Psicología, 10 (2), 239-252. Morales, G., Pichardo, A., Arroyo, R., Canales, C., Silva, H., \& Carpio, C. (2005). Evaluación del aprendizaje de la práctica científica. En C. Carpio y J. J. Irigoyen (Eds.) Psicología y educación. Aportaciones desde la teoría de la conducta (pp. 33-50). México: UNAM. 
Pacheco, V. (2010). ¿Se enseña a escribir a los universitarios? Análisis y propuesta desde la teoría de la conducta. México: UNAM FES Iztacala.

Pacheco, V., Ramírez, L., Palestina, L., \& Salazar, M. (2007). Una aproximación al análisis funcional de la relación entre las conductas de leer y escribir en estudiantes de psicología. En J. J. Irigoyen, M. Jiménez \& K. Acuña (Editores), Enseñanza, aprendizaje y evaluación. Una aproximación a la pedagogía de las ciencias (pp. 247-275). México: UniSon.

Padilla, M. A., Fuentes, N., \& Pacheco, V. (en dictamen). Análisis de los efectos de un entrenamiento correctivo en la elaboración y justificación de preguntas de investigación. Revista Colombiana de Psicología.

Padilla, M.A., \& González, J. (2013, octubre). Effects of varying the characteristics of referential texts on reading and writing scientific texts. Ponencia presentada en la ABAI's 7th International Conference, Mérida, Yucatán, México.

Padilla, M. A., Solórzano, W. G., \& Pacheco, V. (2009). The effects of text analysis on drafting and justifying research questions. Electronic Journal of Research in Educational Psychology, 7 (17), 77-102.

Padilla, M. A., Suro, A. L., \& Tamayo, J. (2010). Efectos de la exposición diferencial a los supuestos de una teoría en la elaboración de preguntas de investigación. Revista Mexicana de Psicología, 27 (2), 247-256.

Padilla, M. A., Tamayo, J., \& González, M. L. (2010). Análisis de la posible relación entre la elaboración de preguntas informales y de investigación. IPyE: Psicología y Educación, 4 (7), 1-21.

Padilla, M.A., Tamayo, J., \& González-Torres,M.L. (en prensa). Does asking pertinent non-academic questions make you a better researcher?: the link between nonacademic and research questions. USA: Nova Science Publishers.

Peña-Correal, T.E., Ordóñez, S., Fonseca, J., \& Fonseca, L. C. (2012). La investigación empírica de la función sustitutiva. En M.A. Padilla \& R. Pérez-Almonacid (Editores), La función sustitutiva referencial: análisis histórico-crítico/avances y perspectivas (pp. 35-100). United States: University Press of the South.

Ribes, E., \& López, F. (1985). Teoría de la conducta, un análisis de campo y paramétrico. México: Trillas.

Ribes, E., Moreno, R., \& Padilla, M. A. (1996). Un análisis funcional de la práctica científica: extensiones de un modelo psicológico. Acta Comportamentalia, 4 (2), 205-235.

Rizo, M. (2004). Hacia una pedagogía de la investigación en comunicología. Reflexiones desde la práctica. En: B. Russi (Ed.) Anuario de la investigación de la comunicación, XI. México: CONEICC-Universidad Intercontinental. 
Sánchez, P. R. (2004). Enseñar a investigar. Una didáctica nueva de la investigación en ciencias sociales y humanas. México: Plaza y Valdés.

Tamayo, J., Padilla, M. A., \& González-Torres, M. L. (2009). Efectos de criterios de logro diferenciales en la elaboración de preguntas informales, en estudiantes de preparatoria, licenciatura y posgrado. Acta Colombiana de Psicología, 12 (1), 27-39.

Viniegra, L. (2002). Un acercamiento a la crítica. Educación y Crítica: el proceso de elaboración del conocimiento. México: Paidós.

Volpato, G., \& Gonçalves-de-Freitas, E. (2003). Desafíos na publicação científica. Pesquisa Odontológica Brasileira, 17 (1), 49-56. 


\section{Apéndice}

\section{Artículos que fueron modificados para emplearse en el estudio}

Guerrero, A. P., \& Ortiz, G. (2007). El papel de la retroalimentación y la ausencia o presencia de instrucciones en la elaboración de descripciones en tareas de discriminación condicional. Acta Colombiana de Psicología, 10 (1), 5-13.

Islas, P. A., \& Flores, C. (2007). Papel de la retroalimentación en la adquisición y transferencia de discriminaciones condicionales en estudiantes universitarios. Enseñanza e Investigación en Psicología, 12 (1), 65-77.

Martínez, H., Ortiz, G., \& González, A. (2007). Efectos diferenciales de instrucciones y consecuencias en ejecuciones de discriminación condicional humana. Psicothema, 19 (1), 14-22.

Villanueva, S., Mateos, R., \& Flores, C. (2008). Efectos del contenido y distribución de la retroalimentación sobre la discriminación condicional de segundo orden. Acta Comportamentalia, 16 (2), 211-221. 
